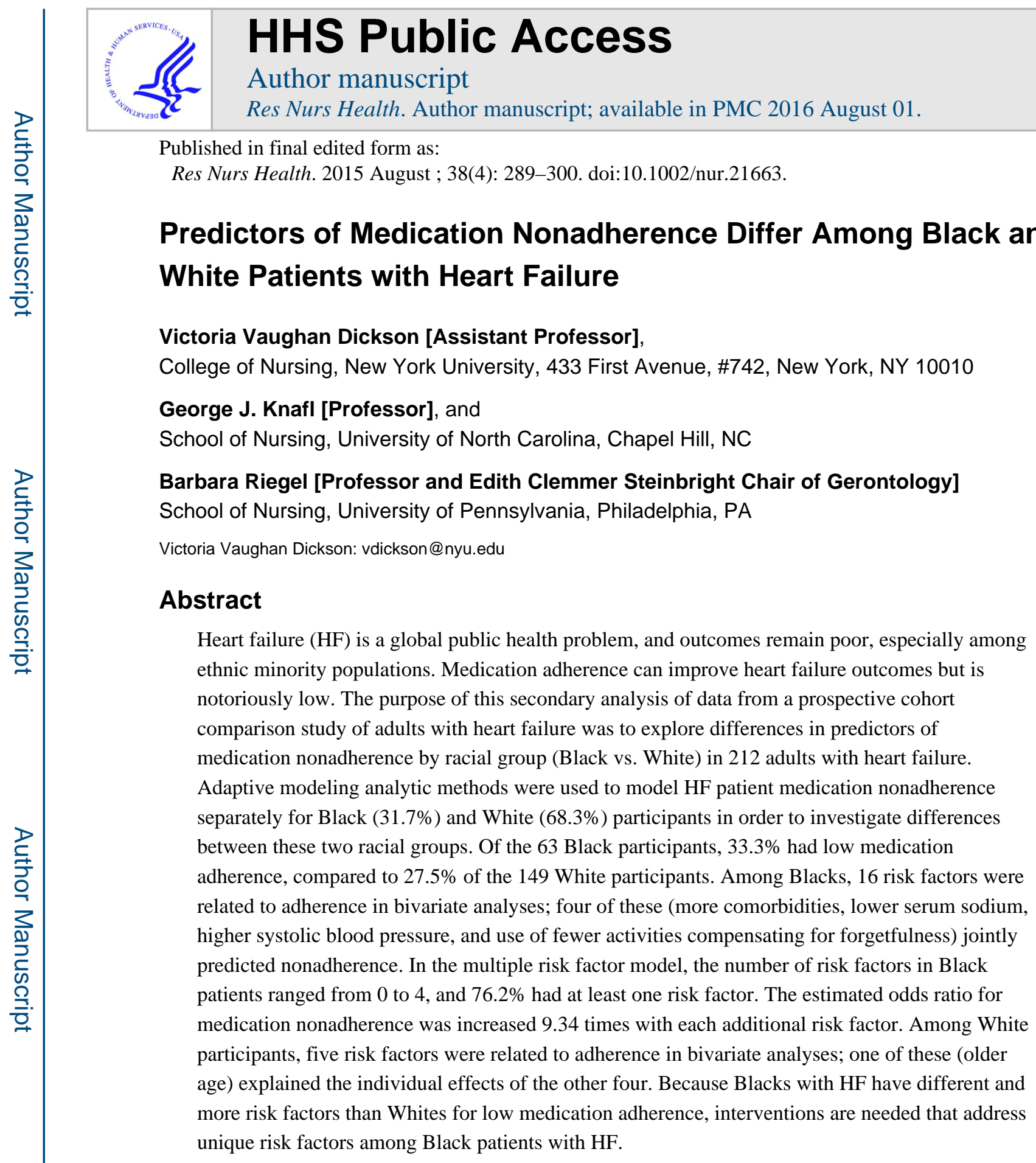

\title{
Keywords
}

Heart failure; medication adherence; self-care; race; disparities

Heart failure (HF) is a global public health issue, with a prevalence of over 5.7 million cases in the United States (US; Mozaffarian et al., 2014) and over 23 million cases worldwide (Bui, Horwich, \& Fonarow, 2011). In the US, the highest prevalence of HF is in Blacks (3.6\%), followed by Whites (2.4\%) and Hispanics (2.3\%; Heidenreich et al., 2013). Overall, 1 in 9 deaths is attributed to HF (Mozaffarian et al., 2014). Ethnic minority patients have a 2.5 times greater risk of HF-related mortality, which is especially pronounced for Black 
males (81.9 national death rate per 100,000 people, compared to 62.1 for White males, and 58.7 for Black females compared to 43.2 for White females; Bahrami et al., 2008).

Heart failure is a major contributor to the cost of health care, predominately because of hospitalizations (Heidenreich et al., 2013). Over the last thirty years, hospitalizations for HF have nearly tripled to more than 1 million in 2006; establishing HF as the most common cause for hospitalization in the Medicare population (Koelling, Johnson, Cody, \& Aaronson, 2005). Compared to Whites, the likelihood of a HF hospitalization is estimated to be 1.5 times greater among Blacks and 1.2 times greater among Hispanics (Brown, Haldeman, Croft, Giles, \& Mensah, 2005).

The reason for this health disparity is complex. Medication nonadherence is one important contributor to hospitalization in patients with HF (Riegel \& Knafl, 2013). In addition, Blacks are more likely than Whites to have diabetes, hypertension and obesity, which increase the risk of $\mathrm{HF}$, complicate management and contribute to poorer outcomes (Schocken et al., 2008). Low socioeconomic status, which is more common in ethnic minority populations, is associated with poor HF outcomes, presumably due to barriers to access and utilization of health care services (Schocken et al., 2008). But surprisingly little research has explored this disparity, so the purpose of this study was to delve further into medication adherence to determine if differences exist that could help to explain the disparity in HF outcomes among White and Black patients.

\section{Medication Adherence in Heart Failure}

Medication adherence is one of a constellation of self-care behaviors (i.e., adherence to treatment regimens, symptom monitoring and symptom management) that can improve HF outcomes. Medication nonadherence is defined as doses not taken or taken incorrectly that jeopardize the therapeutic outcome (Nichols-English \& Poirier, 2000). Nonadherence is estimated at $40 \%$ to $60 \%$ in patients with HF (Wu, Moser, Lennie, \& Burkhart, 2008). This is particularly concerning because medication adherence has been shown to decrease hospitalization (Riegel \& Knafl, 2013), decrease the healthcare costs associated with HF (Wald \& Law, 2003), and improve survival (Wu, Moser, Chung, \& Lennie, 2008a).

In an analysis of Medicare patients with HF, Zhang and Baik (2014) found that in comparison to White patients, ethnic minority populations, including Black and Hispanic patients, were more likely to be nonadherent to common HF medications (i.e., beta-blockers, angiotensin-converting enzyme [ACE] inhibitors, and diuretics), which helps to explain their increased readmission rates, longer hospital stays and increased mortality (Bagchi, Esposito, Kim, Verdier, \& Bencio, 2007; Wu, Moser, Chung, et al., 2008a).

\section{Predictors of Nonadherence}

The reason for differences in medication adherence among White and Black patients with $\mathrm{HF}$ is not entirely clear. Predictors of medication nonadherence include socioeconomic factors (e.g., income), individual-level factors (e.g., race/ethnicity, age, social support), condition-related factors (e.g., comorbid conditions, depression), and provider- or healthcare system-related factors (e.g., provider satisfaction, access; Sabate, 2003). Other reasons 
include forgetfulness (Riegel et al., 2012), perceived lack of benefit (Wu, Moser, Chung, \& Lennie, 2008b), and cost (Van der Wal \& Jaarsma, 2008). Whether the influence of these factors differs between White and Black patients with HF has not been explored.

\section{Research to Date}

We have conducted a series of studies exploring predictors of medication adherence in $\mathrm{HF}$ patients. In the first two analyses, we used the World Health Organization dimensions of adherence (socioeconomic, condition, therapy, patient, and healthcare system) to focus our exploration of contributors to nonadherence (Riegel \& Knafl, 2013; Riegel et al., 2012). In the third analysis, we tested a wide range of clinical and patient-related variables (demographic, social support, self-care, symptoms, and cognition) to avoid omitted variable bias (Knafl \& Riegel, 2014). In a subsequent study we found that Blacks had poorer medication adherence than Whites (Dickson, Knafl, \& Riegel, 2015). Therefore, we sought to examine how predictors of medication nonadherence differ between White and Black patients with HF in order to understand where to focus future interventions aimed at improving medication adherence in those patients most vulnerable to poor HF outcomes.

Specifically, this study addressed these research questions:

1. What factors put Black patients with $\mathrm{HF}$ at risk for medication nonadherence?

2. What factors put White patients with $\mathrm{HF}$ at risk for medication nonadherence?

3. How do these factors differ among Black and White patients with HF?

\section{Methods}

\section{Parent Study}

The methods used in the larger study have been described in other reports (Riegel \& Knafl, 2013; Riegel et al., 2012). In brief, between 2007 and 2009, 280 adults living in the northeastern United States with chronic HF were enrolled into a prospective cohort comparison study. A total of 242 adults completed the study.

Cohorts were purposefully varied on the dimensions of cognition, tested with a neuropsychological battery (Riegel et al., 2011) and daytime sleepiness assessed with the Epworth Sleepiness Scale (Johns, 1992). Inclusion criteria specified that participants have a confirmed diagnosis of HF of any type, fluency in English, and visual, hearing and health literacy sufficient to actively participate in all testing. All participants met a minimum level of cognition screened with the Telephone Interview of Cognitive Status (TICS); anyone with a score $<24$ was excluded. Further exclusion criteria including major depression, renal dialysis, recent history of serious drug or alcohol abuse, imminent death due to terminal illness, and working at night because of the emphasis on exploring sleepiness as a predictor of self-care.

The instruments were administered and other data were collected in a quiet setting in the home by trained research assistants. Institutional Review Board approval was obtained for the study and every participant provided written informed consent. 
Adherence-The Medication Event Monitoring System (MEMS ${ }^{\mathrm{TM}}$; MVW Switzerland Ltd., Sion, Switzerland) attached to a bottle of medication was used to collect medication adherence data objectively from individual patients. The device records the date and time of all bottle openings. MEMS data were collected on one medication scheduled to be taken at a fixed time, preferably more than once daily. Most patients were willing to use the device for one of their cardiac medications (beta blocker 56.7\%, angiotensin-converting enzyme inhibitor $15.2 \%$ ), but a wide variety of medicines was monitored. A single medication was monitored based on the observation of Cramer et al (1995) that patients usually took doses of all their medications simultaneously. That is, if a patient is likely to forget one medicine, chances are that he will forget all of them.

Participants were fully informed that the MEMS device captured their medication-taking behavior in a computer chip. They were instructed on how to integrate the device into their daily routine, even if they routinely used a pill box. MEMS data were collected over the entire 6months of the study and downloaded at 3 and 6 months. Participants kept a study diary for the MEMS in which they could note openings that were accidental or for another purpose (e.g. to fill the container). These deviations were corrected before analysis.

Demographic and clinical variables-Sociodemographic variables, including race, were self-reported. Clinical characteristics such as left ventricular ejection fraction, laboratory values, and the Charlson Comorbidity Index (Charlson, Pompei, Ales, \& MacKenzie, 1987) were abstracted from the medical record by registered nurses. Blood pressure was measured during the home visit using calibrated equipment. Pulse rate was measured at the same time.

Depression-Depression was measured using the Patient Health Questionnaire (PHQ-9; Kroenke, Spitzer, \& Williams, 2001), a reliable and well-validated measure used frequently in HF studies (Masoudi et al., 2004; Sullivan, Newton, Hecht, Russo, \& Spertus, 2004). The PHQ-9 had a sensitivity of $88 \%$ and a specificity of $88 \%$ for detecting major depression when a score of 10 was compared to professional interviews as the criterion standard (Kroenke et al., 2001).

Heart failure self-care-Self-care was measured using the Self-Care of Heart Failure Index (SCHFI V6.2), a 22- item measure of self-care maintenance, management, and confidence (Riegel, Lee, Dickson, \& Carlson, 2009). Construct validity was demonstrated in a sample of $659 \mathrm{HF}$ patients. When internal consistency was tested by factor score determinacy, all coefficients were $>.70$, with moderate to high correlations over time in testretest reliability testing (Barbaranelli, Lee, Vellone, \& Riegel, 2014).

Social support-Social support was measured using the Multidimensional Scale of Perceived Social Support (MSPSS). The 12-item MSPSS measures perceived adequacy of social support from three sources using a 7-point likert scale. Construct validity was demonstrated in a sample of 275 adults and confirmed in subsequent populations (Zimet, 1988; Zimet, Dahlem, Zimet, \& Farley, 1994). Factor analysis confirmed a three-subscale 
structure of the MSPSS: family, friends and significant others (Zimet et al., 1990). The MSPSS has strong alpha coefficients in HF populations (.87 to .91; Dickson, Deatrick, \& Riegel, 2008; Dickson, McCarthy, Howe, Schipper, \& Katz, 2013). In this study, Cronbach alphas for the subscales were very strong $(.92, .93$ and .88$)$; the total scale alpha was .90 .

Compensatory activities-The Compensatory Activities Survey was investigatordesigned to assess methods used to compensate for feeling confused or forgetful. Participants were asked to indicate how often over the past week they used the following five compensatory activities identified from the published literature: 1) keep lists or notes to yourself, 2) get help from your wife/husband, children, neighbor, or others, 3) associate something you want to remember with something else that you already know or remember, 4) visualize the thing you want to remember, 5) repeat new things to yourself. An additional question asked about other techniques used. Each item was scored never, rarely, sometimes, often, frequently or always. For the final "other" activity, nothing else was an additional choice. The number of items endorsed was used as the score. The Cronbach alpha for this survey was .65.

Cognition-Cognition was assessed using a neuropsychological battery of four tests used commonly in patients with HF (Alosco, Spitznagel, Josephson, Hughes, \& Gunstad, 2015; Miller et al., 2012) that measured attention, processing speed, working memory, and shortterm memory. The Digit Symbol Substitution Test (DSST) is a timed test in which numbers are matched with different nonsense symbols. The DSST discriminates age cohorts (Joy et al., 2003) and is sensitive to subtle neurocognitive impairment (Hinton-Bayre, Geffen, \& McFarland, 1997). A poor working memory slows performance and accuracy. The number completed in 120 seconds, the number correctly matched, and percent correct were used in analysis. Test developers reported test-retest reliability of 0.86 (Gregory, 1999).

The Probed Memory Recall (PMR) test is a free recall measure of verbal learning and retention. Four word pairs are studied for 30 seconds. After a 10-minute delay, one word in each pair (in a different order) is presented. The task is to recall all four of the paired words within one minute. Recall of only two of four words suggests that memory is impaired (Dinges, Kribbs, Bates, \& Carlin, 1993).

The Trail Making Test is a widely used and sensitive measure of attention and cognitive flexibility, a component of executive function (Reitan, 1955), and has shown strong testretest reliability (.86 to .94; Wagner, Helmreich, Dahmen, Lieb, \& Tadic, 2011). In patients with HF, the Trail Making Test has demonstrated relationships to areas of brain pathology as assessed by magnetic resonance imaging (Serber et al., 2008). In the Trail Making Test Part A, subjects connect randomly numbered and ordered circles, using a pencil, as fast as possible in serial order. In Part B, on another worksheet, they are asked to connect the same number of consecutively numbered and lettered circles by alternating between numbers and letters.

The Letter Number Sequencing subtest of the Wechsler Adult Intelligence Scale-Third Edition (WAIS III) is primarily a measure of auditory working memory and attention, although it also measures executive function. In this task, participants sequentially order a 
series of numbers and letters that have been orally presented in a specified random order. They must first remember the numbers and letters and then reorganize them into ascending or alphabetical order. Slowed processing speed, inability to remember earlier responses, and reduced capacity to ignore irrelevant information all contribute to decrements in performance. The internal consistency coefficient is .82 (Silva, 2008) and test-retest reliability is .75 (Alosco et al., 2015).

\section{Data Analysis}

For this analysis the variables were organized into six categories: 1) demographics (e.g., age, gender), 2) social support (e.g., marital status, Multidimensional Scale of Perceived Social Support score), 3) clinical (e.g., comorbidities, serum sodium), 4) self-care (e.g., Self-Care of Heart Failure Index, HF knowledge), 5) symptoms (e.g., NYHA class, depression), and 6) cognition (e.g., DSST, Compensatory Activities Survey).

Adaptive modeling of adherence outcome-In our first paper using the MEMS data, standard summary measures of the MEMS data were used, for example, percentage of prescribed doses that were taken (\% PDT; Riegel et al., 2012). Subsequently, adaptive modeling methods (Knafl et al., 2010) were used to analyze available MEMS data more fully (Riegel \& Knafl, 2013). These methods were used to identify individual-patient adherence patterns represented by possibly nonlinear mean adherence and adherence variability curves over time. The adherence patterns were normalized by prescribed rates so that the ideal pattern had constant mean adherence of 1 and constant adherence variability of 0 (although no patients had adherence variability of 0 ). Then, these individual-patient adherence patterns were clustered into adherence types or groups of participants with similar adherence patterns.

Seven adherence types were identified, but hospitalization within 6 months was best predicted by combining the seven types into the dichotomous predictor of poor adherence (i.e., three of the clusters with adherence deteriorating to zero adherence or nearly zero over time) versus better adherence (i.e., four clusters with moderate to high adherence over the entire measurement period). This adaptively-determined dichotomized outcome served as the nonadherence outcome for the analyses reported in this article.

Model development of predictors of nonadherence-Logistic regression models for medication nonadherence were generated using adaptive modeling methods (Knafl et al., 2010). These methods use $k$-fold likelihood cross-validation (LCV) scores to evaluate and compare models, with larger LCV scores indicating better models. Observations are separated into $k$ disjoint subsets, called folds, using random numbers. For each fold, parameter estimates for a given model are computed using the data in all the other folds. These deleted parameter estimates are then used to compute likelihoods for the folds, which are then normalized by the sample size and combined into the LCV score for the associated model. For reported analyses, LCV scores are based on 10 folds and on likelihoods for logistic regression models.

LCV scores for two models can be compared with LCV ratio tests, analogous to likelihood ratio tests, but conducted using the percent decrease for the smaller LCV score compared to 
the larger LCV score. LCV-based comparisons provide more objective assessments of effects of individual predictors in comparison to significance tests of slopes for those predictors. Moreover, they can be more conservative, identifying predictors as having insubstantial effects when the associated test for zero slope is significant (see the Results for examples), and so are similar in effect to multiple comparisons procedures.

Each data set has an associated cutoff for a substantial (distinct or significant) percent decrease in LCV scores that depends on the number of observations in that data set. A percent decrease greater than this cutoff indicates that the model with the smaller LCV score is improved upon substantially by the model with the larger LCV score. When the model with the smaller LCV score is simpler, it is preferable. A percent decrease less than or equal to this cutoff indicates that the model with the smaller LCV score is a competitive alternative to the model with the larger LCV score. For example, when the constant model is compared to a model based on a single risk factor, a percent decrease in LCV scores less than the cutoff for the data indicates that this risk factor has an insubstantial effect on medication nonadherence. In the present analysis, cutoffs for a substantial percent decrease in the LCV scores were $3.00 \%$ for Blacks and $1.28 \%$ for Whites.

Separate analyses for the Black and White subgroups were chosen over moderation analyses of the combined data. When $\mathrm{x}$ is a possible predictor, a moderation analysis would include both the predictor $\mathrm{x}$ and its interaction with being Black. This approach assumes that the predictor has an effect for Whites that differs from the effect for Blacks. However, it is possible that $\mathrm{x}$ has an effect only for Blacks and not for Whites, or vice versa. Subgroup analyses were chosen because they allowed for different predictors for Blacks and Whites.

Dichotomous risk factors for medication nonadherence were identified separately for Black and White participants using the following process: 1) Bivariate models were used to identify risk factors with an odds ratio $(O R)>1$ for medication nonadherence. 2) Identified risk factors with significant $(\mathrm{I}<.05)$ slopes were combined adaptively into a multiple risk factors model. 3) The adaptive multiple risk factors model was used to generate the associated risk index model for medication nonadherence, based on the count of that model's risk factors. Interactions between risk factors were not considered in these subgroup analyses (as they were in analyses of the full data; Knafl \& Riegel, 2014) because meaningful interaction results were unlikely given the modest subgroup sample sizes.

In step 1, categorical variables were used unchanged. In subsequent analysis steps, the predictors, whether ordinal or continuous, were dichotomized as follows. A predictor's observed values were split into two sets, using each of those observed values as a threshold for low versus high ranges of values. Only cases with at least $10 \%$ of the observations in each range of values were considered. The selected threshold was the one generating the best LCV score for predicting medication nonadherence. Cases with values producing an $O R$ $>1$ were treated as having the risk factor. Observations with missing values were conservatively assigned to the non-risk factor range of values. Information can be lost when a predictor is categorized into two levels, so LCV scores for categorized and uncategorized predictors were compared. Comparisons were limited to predictors with no missing values, because the compared LCV scores would have been computed for the same set of data. 
In step 2, a model was selected using the adaptive modeling process of Knafl et al. (2010). Risk factors under consideration for inclusion in the model were added systematically to the model using LCV scores to decide which risk factor to add next. Then this expanded model was contracted, removing any extraneous risk factors. LCV ratio tests were used to decide whether to continue contracting the model or stop the contraction.

In step 3, the count of the number of risk factors in the adaptively generated multiple risk factors model was used as a risk index variable for predicting medication nonadherence.

\section{Results}

\section{Sample}

Usable MEMS data were available for 218 (90.1\%) of the 242 participants who completed the parent study. Of these 218 participants with MEMS data, 149 (68.3\%) were White and $69(31.7 \%)$ were non-White. Of those who were non-White, the vast majority was Black (63 or $91.3 \%$ out of 69$)$; 4 were Hispanic and 2 were American Indian. Therefore, we focused our analysis on the 212 White and Black participants.

Table 1 provides a comparison of Blacks and Whites on selected demographic characteristics. Black participants were significantly more likely than White participants to be retired, unemployed, or disabled, to report insufficient income, to have government or no insurance, and to be single, divorced, separated, or widowed. Black participants had significantly fewer years of education on average.

Table 2 provides a comparison of Blacks and Whites on selected clinical variables. Blacks were significantly more likely to perceive their general health as poor and to have severely limited functioning (NYHA functional class IV). Black participants had significantly more comorbid conditions, took more medications, and took more doses of those medications each day.

Low adherence based on MEMS is often defined as percent doses taken (\% PDT) $<88 \%$ (Wu et al., 2009). A total of 87 (41.0\%) of the 212 participants had \% PDT $\geq 88 \%$, with 27 (28.7\%) of these hospitalized in 6 months. The remaining 63 (29.7\%) participants had $\%$ PDT $<88 \%$, and better adherence as determined adaptively, with 18 (28.6\%) hospitalized in 6 months. A total of 62 (29.2\%) of the 212 participants had poor adaptively measured adherence, with 35 of these 62 (56.5\%) of these hospitalized within six months. All these participants also had poor adherence when traditionally measured as \% PDT $<88 \%$. The hospitalization rates were almost the same for these latter two groups, demonstrating that poor adherence determined adaptively is a more sensitive indicator than \% PDT $<88 \%$. Consequently, \% PDT was not considered in further analyses.

When examined using \%PDT, Black participants were significantly more likely than White participants to have this type of poor adherence ( $p=.001 ; 76.2 \%$ vs. $51.7 \%)$. However, when poor adherence was determined adaptively, Black participants were no more likely than Whites to have poor adherence $(p=.395 ; 33.3 \%$ vs. $27.5 \%)$. 


\section{Risk Factors for Medication Nonadherence for Black Participants}

Table 3 contains results for individual risk factor analyses of medication nonadherence (as determined adaptively) for Black participants. There were 16 individually significant ( $\mathrm{p} \leq$ 05 ) risk factors. The constant model generated a substantial percent decrease in the LCV scores (greater than the previously determined cutoff of 3.00\%) for 10 of these 16 risk factors (LCV scores not reported), indicating that LCV ratio tests can generate conservative results. The individual risk factor model based on more comorbidities had the best LCV score of 0.54732 among all the individual risk factor models of Table 3.

Of the 15 risk factors of Table 3, 10 could be analyzed as continuous or ordinal predictors (not counting living alone) as well as risk-factor categories and had no missing values, so these 10 risk-factor models could be compared to models linear in the associated predictors, to assess the impact of categorizing these variables. For all 10 of these predictors, the categorized risk-factor model generated the better LCV score than the associated linearpredictor model. Moreover, for five of these predictors (blood urea nitrogen, creatinine, serum sodium, PHQ total score, and DSST score), the linear-predictor model generated a substantially lower percent decrease in the LCV score. This finding indicated that adaptively categorizing these variables into two-level risk factors provided substantially better predictions of poor adherence than that obtained with standard linear-predictor modeling.

Table 4 contains results for the adaptive multiple risk factors model based on the 16 significant individual risk factors of Table 3 . This model included four risk factors jointly predicting poor adherence: more comorbidities, lower serum sodium, higher systolic blood pressure, and fewer methods of compensating for forgetfulness. This model had a LCV score of 0.61437 , which was a substantial improvement over the individual risk factor model with the best LCV, with a substantial percent decrease of $10.91 \%$. Consequently, the multiple risk factors model for Black participants provided a substantial improvement over all the individual risk factor models. This model had a $c$-index (the same as the area under the receiver-operating characteristics [ROC] curve) of 0.86 , which is considered excellent discrimination (Hosmer \& Lemeshow, 2000). The Nagelkerke $R^{2}$ was $50.4 \%$.

Numbers of risk factors from the multiple risk factor model for the 63 Black patients ranged from 0 to 4, with 15 (23.8\%) patients having none of the risk factors, 27 (42.9\%) patients having 1, $17(27.0 \%)$ having 2, $3(4.8 \%)$ patients having 3 , and $1(1.6 \%)$ patient having all 4 risk factors. For the 15 patients with 0 risk factors, none were nonadherent; for the 27 patients with 1 risk factor, $22.2 \%$ were nonadherent; for the 17 patients with 2 risk factors, $64.7 \%$ were nonadherent; for the 4 patients with 3 or 4 risk factors, $100 \%$ were nonadherent to the medication regimen.

To assess the possibility of collinearity between these four risk factors, logistic regression models were computed predicting each of them as a function of the other three. The largest Nagelkerke $R^{2}$ value for these models was $2.2 \%$. Thus, collinearity was not a problem for the multiple risk factors model.

When the number of these 4 risk factors, treated as a risk index, was used as a single predictor of nonadherence, this risk index model had a LCV score of 0.64523 and improved 
substantially on the multiple risk factors model, with a percent decrease in the LCV score of $4.78 \%$. The $c$-index for this risk index model was also excellent at .85 . The estimated $O R$ for medication nonadherence with each unit increase in the risk index count variable was 9.34 (95\% CI 3.04 to $28.7 ; p<.001$ ). That is, the odds of nonadherence increased by 9.34 times with each additional risk factor.

\section{Risk Factors for Medication Nonadherence for White Participants}

Table 5 contains results for individual risk factor analyses of medication nonadherence for White participants. There were 5 significant $(p \leq 05)$ individual risk factors (age, comorbidities, months since HF diagnosis, total medication doses, cognition). The constant model generated a substantial percent decrease in the LCV scores (greater than the cutoff of $1.28 \%$ for the White data) for $2(40.0 \%)$ of these 5 risk factors (LCV scores not reported), indicating once again that $\mathrm{LCV}$ ratio tests can generate conservative results.

Of the five risk factors of Table 5 based on continuous or ordinal predictors, three of them had no missing values and could be compared to categorized risk factors. In all three cases, the risk-factor model generated the better LCV score than the associated linear-predictor model. For two (66.7\%) of these predictors (age and TMTB score), the linear-predictor model generated a substantially lower percent decrease in the LCV score, indicating that adaptively categorizing these variables into two-level risk factors provided substantially better predictions of poor adherence than was obtainable through standard linear-predictor modeling.

The adaptive multiple risk factors model based on the five individually significant risk factors of Table 5 identified only one risk factor: older age, which was the individual risk factor model with the best LCV score of 0.56240 among all the individual risk factor models. With only one risk factor, collinearity or a risk index model could not be considered. The $c$-index for this singleton predictor model was .62, less than the cutoff of . 70 for acceptable discrimination (Hosmer \& Lemeshow, 2000). The Nagelkerke $R^{2}$ was $7.4 \%$.

\section{Discussion}

Although others have reported differences in medication adherence by race/ethnicity or identified the predictors of medication adherence among patients with HF in general, this analysis is the first, to our knowledge, to differentiate the predictors of adherence by racial group. Our findings that Blacks have more and different risk factors for poor medication adherence is important because it provides specific guidance on where to focus interventions aimed at improving medication adherence and related HF outcomes. We found that Blacks had four risk factors that jointly predicted nonadherence (more comorbidities, lower serum sodium, higher systolic blood pressure, and use of fewer activities compensating for forgetfulness). Among White participants, only one risk factor (older age) explained the individual effects of the other four, which is consistent with evidence that medication nonadherence is common in the elderly (MacLaughlin et al., 2005). 
Comorbidity was identified as one of the four major risk factors among Blacks. Comorbidity is extremely common in patients with HF (Braunstein et al., 2003) and adds complexity to the medication regimen required of patients, in part due to the number of medications, drug interactions, and side effects (Mastromarino et al., 2014). Multiple chronic conditions have been shown to complicate self-care when patient care is fragmented (Dickson, Buck, \& Riegel, 2011). When faced with uncertainty about medication or unpleasant medication side effects, patients with HF described the influence of self-efficacy on their daily self-care, including decisions about which medications to take (Dickson, Buck, \& Riegel, 2013). Patients with multiple chronic conditions also experience more functional limitations that make daily activities difficult, such as traveling to pick up medicines, remembering to take them, and symptom recognition and management (Bakas, Pressler, Johnson, Nauser, \& Shaneyfelt, 2006). Because medication adherence is essential to HF outcomes (Riegel \& Knafl, 2013; Wu, Moser, Chung, et al., 2008a), our results suggest that Blacks may be more vulnerable to the burden of multiple chronic conditions than Whites. Focused efforts to integrate care and interventions to facilitate medication adherence are essential.

In Whites both comorbidity and cognition were individual determinants of nonadherence, but their influence on adherence was explained away by older age in the final analysis. That is, in Whites, nonadherence was related to age in a strong enough way that the effects of comorbidity and cognition on adherence were no longer of substance. This finding was surprising to us because it suggests that there is an element of aging itself, beyond comorbidity and cognition, that is influencing nonadherence. Further research is needed to determine the aspects of aging that are associated with medication nonadherence.

A lower level of serum sodium was a predictor of nonadherence in Blacks. At a cut-point of $\leq 139 \mathrm{meq} / \mathrm{L}$, these serum sodium levels did not qualify as true hyponatremia. However, because fluid retention stimulates atrial natriuretic peptide secretion and the loss of sodium, a lower level of serum sodium is an indicator of the severity of cardiac dysfunction (Gheorghiade et al., 2007). Thus, these results suggest that either the sicker Black patients are those who are most likely to be nonadherent, or those who are nonadherent are most likely to become sicker. To the best of our knowledge, this is the first study to demonstrate a relationship between serum sodium and poor medication adherence.

Interestingly, higher systolic BP was one of the four joint predictors of poor medication adherence in Blacks but not in Whites. Previously we reported that Blacks were more likely than Whites to have hypertension and to be prescribed isosorbide dinitrate and diuretics (Dickson, Knafl, \& Riegel, 2015). Guidelines recommend that the pharmacologic regimen for patients with HF include angiotensin-converting-enzyme (ACE) inhibitors, beta blockers and angiotensin receptor blockers (Yancy et al., 2013), all medications that lower BP. In fact, poor blood pressure control is commonly blamed on medication nonadherence (Hayen, Bell, Glasziou, Neal, \& Irwig, 2010), and elevated systolic BP is associated with poor medication adherence (Braverman \& Dedier, 2009; Knafl, Schoenthaler, \& Ogedegbe, 2012). Therefore, our finding that systolic BP is higher among Blacks may be a consequence of poor medication adherence rather than a cause of nonadherence. 
The final factor significantly associated with nonadherence in Blacks was the use of fewer methods to compensate for feeling confused or forgetful. We used a new measure of the methods used by patients to compensate for such feelings. Other researchers have found that older adults use internal and external strategies to remember to take their medications including visual cues, consistent routines and physical objects (e.g., pill boxes; Gould, McDonald-Miszczak, \& King, 1997). Such compensatory strategies were associated with higher education, more medications, and self-efficacy to take medications, but differences by racial group were not explored (Boron, Rogers, \& Fisk, 2013). Our finding that lack of such methods was a significant risk factor for Blacks requires further exploration. How individuals develop or adapt such methods, especially related to medication adherence, may help inform future interventions.

\section{Limitations}

Interactions were not considered because subgroup sample sizes were unlikely to be large enough for interaction analysis. Further, our sample was English-speaking and fairly welleducated. Further research is needed in an adequately powered sample of persons of color that includes non-English-speaking individuals and those with low literacy, groups which were not adequately represented in the present study. In this study, measurement of adherence was limited to one medication, and new data suggest that some subtle differences exist in the adherence to various HF medicines (Viana et al., 2014). These data were collected before this article was published, but future studies should monitor a single drug class in all participants or preferably all the major drugs in the regimen. A strength of the study was the use of objective measures of medication adherence using the MEMS rather than relying on self-report or pharmacy data to calculate medication adherence. Self-report has been shown to be unreliable, so our method of measuring medication adherence strengthens our conclusions.

\section{Conclusions}

Although similar patterns of nonadherence have been found by others (Zhang \& Baik, 2014) our findings suggest that the issue of medication adherence is exceedingly complex for many persons with HF, especially Blacks. Many of these patients were taking complex medication regimens, with substantial numbers of medications. An individualized approach may be needed in order to address the health disparities associated with medication nonadherence. Specifically, clinicians should be sensitive to those factors (e.g., multiple comorbid conditions) that may place ethnic minorities at increased risk for poor adherence. Further research is needed to develop and test interventions that help Black patients with HF to manage multiple medications required by multiple chronic conditions. These results also reinforce the need to address methods of compensating for forgetfulness when teaching Blacks about their medication regimens.

\section{Acknowledgments}

This work was funded in part by a grant from the National Heart, Lung \& Blood Institute (R01 HL084394-01A1) and by the Philadelphia Veterans Affairs Medical Center, VISN 4 Mental Illness Research, Education, and Clinical Center (MIREC). The authors gratefully acknowledge Megan Patey for her assistance with preparation of the data for analysis. 


\section{References}

Alosco ML, Spitznagel MB, Josephson R, Hughes J, Gunstad J. COPD is associated with cognitive dysfunction and poor physical fitness in heart failure. Heart \& Lung. 2015; 44:21-26.10.1016/ j.hrtlng.2014.09.002 [PubMed: 25308768]

Bagchi A, Esposito D, Kim M, Verdier J, Bencio D. Utilization of, and adherence to, drug therapy among Medicaid beneficiaries with congestive heart failure. Clinical Therapeutics. 2007; 29:17711783.10.1016/j.clinthera.2007.08.015 [PubMed: 17919558]

Bahrami H, Kronmal R, Bluemke DA, Olson J, Shea S, Liu K, Lima JA. Differences in the incidence of congestive heart failure by ethnicity: the multi-ethnic study of atherosclerosis. Archivesof Internal Medicine. 2008; 168:2138-2145.10.1001/archinte.168.19.2138

Bakas T, Pressler S, Johnson E, Nauser J, Shaneyfelt T. Family caregiving in heart failure. Nursing Research. 2006; 55:180-188. [PubMed: 16708042]

Barbaranelli C, Lee C, Vellone E, Riegel B. Dimensionality and reliability the Self-Care of Heart Failure Index scales: Further evidence from Confirmatory Factor Analysis. Research in Nursing \& Health. 2014; 37:524-537.10.1002/nur.21623 [PubMed: 25324013]

Boron JB, Rogers WA, Fisk AD. Everyday memory strategies for medication adherence. Geriatric Nursing. 2013; 34:395-401.10.1016/j.gerinurse.2013.05.010 [PubMed: 23810198]

Braunstein J, Anderson G, Gerstenblith G, Weller W, Niefeld M, Herbert R, Wu A. Noncardiac comorbidity increases preventable hospitalizations and mortality among Medicare beneficiaries with chronic heart failure. Journal of the American College of Cardiology. 2003; 42:1226-1233. [PubMed: 14522486]

Braverman J, Dedier J. Predictors of medication adherence for African American patients diagnosed with hypertension. Ethnicty \& Disease. 2009; 19:396-400.

Brown DW, Haldeman GA, Croft JB, Giles WH, Mensah GA. Racial or ethnic differences in hospitalization for heart failure among elderly adults: Medicare, 1990 to 2000. American Heart Journal. 2005; 150:448-454.10.1016/j.ahj.2004.11.010 [PubMed: 16169322]

Bui AL, Horwich TB, Fonarow GC. Epidemiology and risk profile of heart failure. Nature Reviews Cardiology. 2011; 8:30-41.10.1038/nrcardio.2010.165 [PubMed: 21060326]

Charlson M, Pompei P, Ales K, MacKenzie C. A new method of classifying prognostic comorbidity in longitudinal studies: development and validation. Journal of Chronic Diseases. 1987; 40:373-383. [PubMed: 3558716]

Dickson V, Buck H, Riegel B. A qualitative meta-analysis of heart failure self-care practices among individuals with multiple comorbid conditions. Journal of Cardiac Failure. 2011; 17:413419.10.1016/j.cardfail.2010.11.011 [PubMed: 21549299]

Dickson V, Buck H, Riegel B. Multiple comorbid conditions challenge heart failure self-care by decreasing self-efficacy. Nursing Research. 2013; 62:2-9.10.1097/NNR.0b013e31827337b3 [PubMed: 23052421]

Dickson V, Deatrick J, Riegel B. A typology of heart failure self-care management in non-elders. European Journal of Cardiovascular Nursing. 2008; 7:171-181.10.1016/j.ejcnurse.2007.11.005 [PubMed: 18178132]

Dickson V, Knafl G, Riegel B. Racial differences in clinical treatment and self-care behaviors of adults with chronic heart failure. Journal of the American Heart Association. 2015; 4:e001561. doi: 10.1161. [PubMed: 25870187]

Dickson V, McCarthy M, Howe A, Schipper J, Katz S. Socio-cultural influences on heart failure selfcare among an ethnic minority black population. Journal of Cardiovascular Nursing. 2013; 28:111-118.10.1097/JCN.0b013e31823db328 [PubMed: 22343210]

Dinges D, Kribbs N, Bates B, Carlin M. A very brief probed-recall memory task: Sensitivity to sleep loss. Sleep Research. 1993; 22:330.

Gheorghiade M, Abraham WT, Albert NM, Gattis Stough W, Greenberg BH, O’Connor CM, Fonarow GC. Relationship between admission serum sodium concentration and clinical outcomes in patients hospitalized for heart failure: An analysis from the OPTIMIZE-HF registry. European Heart Journal. 2007; 28:980-988.10.1093/eurheartj/ehl542 [PubMed: 17309900] 
Gould ON, McDonald-Miszczak L, King B. Metacognition and medication adherence: how do older adults remember? Experimental Aging Research. 1997; 23:315-342.10.1080/03610739708254034 [PubMed: 9352290]

Gregory, R. Foundations of intellectual assessment: The WAIS-III and other tests in clinical practice. Boston: Allyn and Bacon; 1999.

Hayen A, Bell K, Glasziou P, Neal B, Irwig L. Monitoring adherence to medication by measuring change in blood pressure. Hypertension. 2010; 56:612-616.10.1161/HYPERTENSIONAHA. 110.153817 [PubMed: 20696980]

Heidenreich P, Albert NM, Allen LA, Bluemke DA, Butler J, Fonarow GC, Trogdon JG. Forecasting the impact of heart failure in the United States: A policy statement from the American Heart Association. Circulation: Heart Failure. 2013; 6:606-619.10.1161/HHF.0b013e318291329a [PubMed: 23616602]

Hinton-Bayre AD, Geffen G, McFarland K. Mild head injury and speed of information processing: a prospective study of professional rugby league players. Journal of Clinical and Experimental Neuropsychology. 1997; 19:275-289. [PubMed: 9240486]

Hosmer, D.; Lemeshow, S. Applied logistic regression. 2. New York: John Wiley \& Sons; 2000.

Johns MW. Reliability and factor analysis of the Epworth Sleepiness Scale. Sleep. 1992; 15:376-381. [PubMed: 1519015]

Joy S, Kaplan E, Fein D. Digit symbol-incidental learning in the WAIS-III: construct validity and clinical significance. Clinical Neuropsychology. 2003; 17:182-194.10.1076/clin.17.2.182.16495

Knafl, G.; Delucchi, K.; Bova, C.; Fennie, K.; Ding, K.; Williams, A. A systematic approach for analyzing electronically monitored adherence data. Hauppauge, NY: Nova Science Publishers; 2010.

Knafl G, Riegel B. What puts heart failure patients at risk for poor medication adherence. Patient Preferences \& Adherence. 2014; 8:1007-1018.10.2147/PPA.S64593

Knafl G, Schoenthaler A, Ogedegbe G. Secondary analysis of electronically monitored medication adherence data for a cohort of hypertensive African-Americans. Patient Preferences \& Adherence. 2012; 6:207-219.10.2147/ppa.s30582

Koelling TM, Johnson ML, Cody RJ, Aaronson KD. Discharge education improves clinical outcomes in patients With chronic heart failure. Circulation. 2005; 111:179-185.10.1161/01.cir. 0000151811.53450.b8 [PubMed: 15642765]

Kroenke K, Spitzer R, Williams J. The PHQ-9: Validity of a brief depression severity measure. Journal of General Internal Medicine. 2001; 16:606-613. [PubMed: 11556941]

Lezak, MD.; Howieson, DB.; Loring, DW. Neuropsychological assessment. 4. New York: Oxford University Press; 2004.

MacLaughlin EJ, Raehl CL, Treadway AK, Sterling TL, Zoller DP, Bond CA. Assessing medication adherence in the elderly: Which tools to use in clinical practice? Drugs \& Aging. 2005; 22:231255. [PubMed: 15813656]

Masoudi F, Rumsfeld J, Havranek E, House J, Peterson E, Krumholz H, Spertus J. Age, functional capacity, and health-related quality of life in patients with heart failure. Journal of Cardiac Failure. 2004; 10:368-373. [PubMed: 15470645]

Mastromarino V, Casenghi M, Testa M, Gabriele E, Coluccia R, Rubattu S, Volpe M. Polypharmacy in heart failure patients. Current Heart Failure Reports. 2014; 11:212-219.10.1007/ s11897-014-0186-8 [PubMed: 24493574]

Miller LA, Spitznagel MB, Alosco ML, Cohen RA, Raz N, Sweet LH, Gunstad J. Cognitive profiles in heart failure: A cluster analytic approach. Journal of Clinical and Experimental Neuropsychology. 2012; 34:509-520.10.1080/13803395.2012.663344 [PubMed: 22375800]

Mozaffarian D, Benjamin EJ, Go AS, Arnett DK, Blaha MJ, Cushman M, Turner MB. Heart disease and stroke statistics-2015 update: A report from the American Heart Association. Circulation. 2014; 131:e29-322.10.1161/cir.0000000000000152 [PubMed: 25520374]

Nichols-English G, Poirier S. Optimizing adherence to pharmaceutical care plans. Journal of the American Pharmaceutical Association. 2000; 40:475-485. [PubMed: 10932456]

Reitan RM. The relation of the trail making test to organic brain damage. Journal of Consulting Psychology. 1955; 19:393-394. [PubMed: 13263471] 
Reitan, RM. Trail Making Test. Tucson, AZ: Reitan Neuropsychological Laboratory; 1992.

Riegel B, Knafl GJ. Electronically monitored medication adherence predicts hospitalization in heart failure patients. Patient Preferences \& Adherence. 2013; 8:1-13.10.2147/PPA.S54520

Riegel B, Lee C, Dickson V, Carlson B. An update on the Self-Care of Heart Failure Index. Journal of Cardiovascular Nursing. 2009; 24:485-497.10.1097/JCN.0b013e3181b4baa0 [PubMed: 19786884]

Riegel B, Lee C, Ratcliffe S, De Geest S, Potashnik S, Patey M, Weintraub W. Predictors of objectively measured medication nonadherence in adults with heart failure. Circulation: Heart Failure. 2012; 5:430-436.10.1161/circheartfailure.111.965152 [PubMed: 22647773]

Riegel B, Moelter S, Ratcliffe S, Pressler SJ, De Geest S, Potashnik S, Goldberg LR. Excessive daytime sleepiness is associated with poor medication adherence in adults with heart failure. Journal of Cardiac Failure. 2011; 17:340-348.10.1016/j.cardfail.2010.11.002 [PubMed: 21440873]

Sabate, E. Adherence to long-term therapies: Evidence for action. Geneva: World Health Organization; 2003.

Schocken D, Benjamin E, Fonarow G, Krumholz H, Levy D, Mensah G, Hong Y. Prevention of heart failure: A scientific statement from the American Heart Association Councils on Epidemiology and Prevention, Clinical Cardiology, Cardiovascular Nursing, and High Blood Pressure Research; Quality of Care and Outcomes Research Interdisciplinary Working Group; and Functional Genomics and Translational Biology Interdisciplinary Working Group. Circulation. 2008; 117:2544-2565.10.1161/circulationaha.107.188965 [PubMed: 18391114]

Serber SL, Kumar R, Woo MA, Macey PM, Fonarow GC, Harper RM. Cognitive test performance and brain pathology. Nursing Research. 2008; 57:75-83.10.1097/01.nnr.0000313483.41541.10 [PubMed: 18347478]

Silva M. Development of the WAIS-III: Brief overview, history and description. Graduate Journal of Counseling Psychology. 2008; 1:117-135.

Sullivan M, Newton K, Hecht J, Russo J, Spertus J. Depression and health status in elderly patients with heart failure: A 6-month prospective study in primary care. American Journal of Geriatric Cardiology. 2004; 13:252-260. [PubMed: 15365288]

Van der Wal M, Jaarsma T. Adherence in heart failure in the elderly: Problem and possible solutions. [Review]. International Journal of Cardiology. 2008; 125:203-208.10.1016/j.ijcard.2007.10.011 [PubMed: 18031843]

Viana M, Laszczynska O, Mendes S, Frioes F, Lourenco P, Bettencourt P, Azevedo A. Medication adherence to specific drug classes in chronic heart failure. Journal of Managed Care Pharmacy. 2014; 20:1018-1026.

Wagner S, Helmreich I, Dahmen N, Lieb K, Tadic A. Reliability of three alternate forms of the Trail Making Tests A and B. Archives in Clinical Neuropsychology. 2011; 26:314-321.10.1093/arclin/ acr024

Wald N, Law M. A strategy to reduce cardiovascular disease by more than $80 \%$. BMJ. 2003; 326(7404):1419. doi: http://dx.doi.org/10.1136/bmj.326.7404.1419. [PubMed: 12829553]

Wechsler, D. WAIS-III: Administration and scoring manual. 3. San Antonio, TX: Psychological Corporation; 1997.

Wu J, Moser D, Chung M, Lennie T. Objectively measured, but not self-reported, medication adherence independently predicts event-free survival in patients with heart failure. Journal of Cardiac Failure. 2008a; 14:203-210.10.1016/j.cardfail.2007.11.005 [PubMed: 18381183]

Wu J, Moser D, Chung M, Lennie T. Predictors of medication adherence using a multidimensional adherence model in patients with heart failure. Journal of Cardiac Failure. 2008b; 14:603614.10.1016/j.cardfail.2008.02.011 [PubMed: 18722327]

Wu J, Moser D, De Jong M, Rayens M, Chung M, Riegel B, Lennie T. Defining an evidence-based cutpoint for medication adherence in heart failure. American Heart Journal. 2009; 157:285291.10.1016/j.ahj.2008.10.001 [PubMed: 19185635]

Wu J, Moser D, Lennie T, Burkhart P. Medication adherence in patients who have heart failure: A review of the literature. Nursing Clinics of North America. 2008; 43:133-153.10.1016/j.cnur. 2007.10.006 [PubMed: 18249229] 
Wu J, Moser D, Lennie T, Peden A, Yu-Chang C, Heo S. Factors influencing medication adherence in patients with heart failure. Heart \& Lung. 2008; 37:8-16.10.1016/j.hrtlng.2007.02.003 [PubMed: 18206522]

Yancy CW, Jessup M, Bozkurt B, Butler J, Casey DE Jr, American College of Cardiology Foundation/ American Heart Association Task Force on Practice, G. 2013 ACCF/AHA guideline for the management of heart failure: A report of the American College of Cardiology Foundation/ American Heart Association Task Force on practice guidelines. Circulation. 2013; 128:e240327.10.1161/CIR.0b013e31829e8776 [PubMed: 23741058]

Zhang Y, Baik S. Race/ethnicity, disability, and medication adherence among Medicare beneficiaries with heart failure. Journal of General Internal Medicine. 2014; 29:602-607.10.1007/ s11606-013-2692-x [PubMed: 24366395]

Zimet G. Multidimensional Scale of Perceived Social Support. Journal of Personality Assessment. $1988 ; 52: 30-41$.

Zimet, G.; Dahlem, N.; Zimet, S.; Farley, G. Multidimensional Scale of Perceived Social Support. In: Fischer, J.; Corcoran, K., editors. Measures for clinical practice: A sourcebook. Volume 2: Adults. 2. New York: Free Press; 1994. p. 393-394.

Zimet G, Powell S, Farley G, Werkman S, Berkoff K. Psychometric characteristics of the Multidimensional Scale of Perceived Social Support. Journal of Personality Assessment. 1990; 55:610-617.10.1080/00223891.1990.9674095 [PubMed: 2280326] 
Dickson et al.
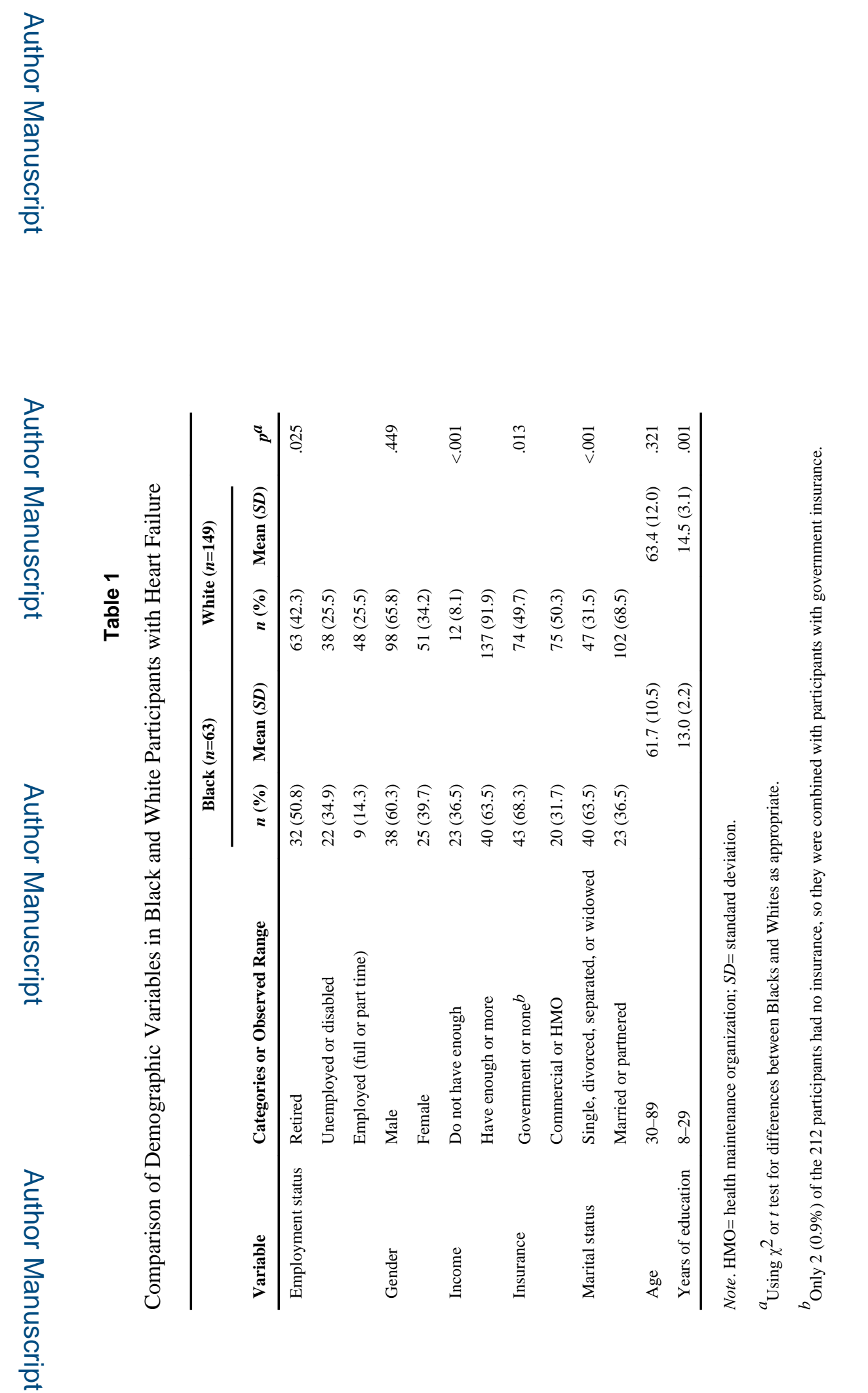

Res Nurs Health. Author manuscript; available in PMC 2016 August 01. 


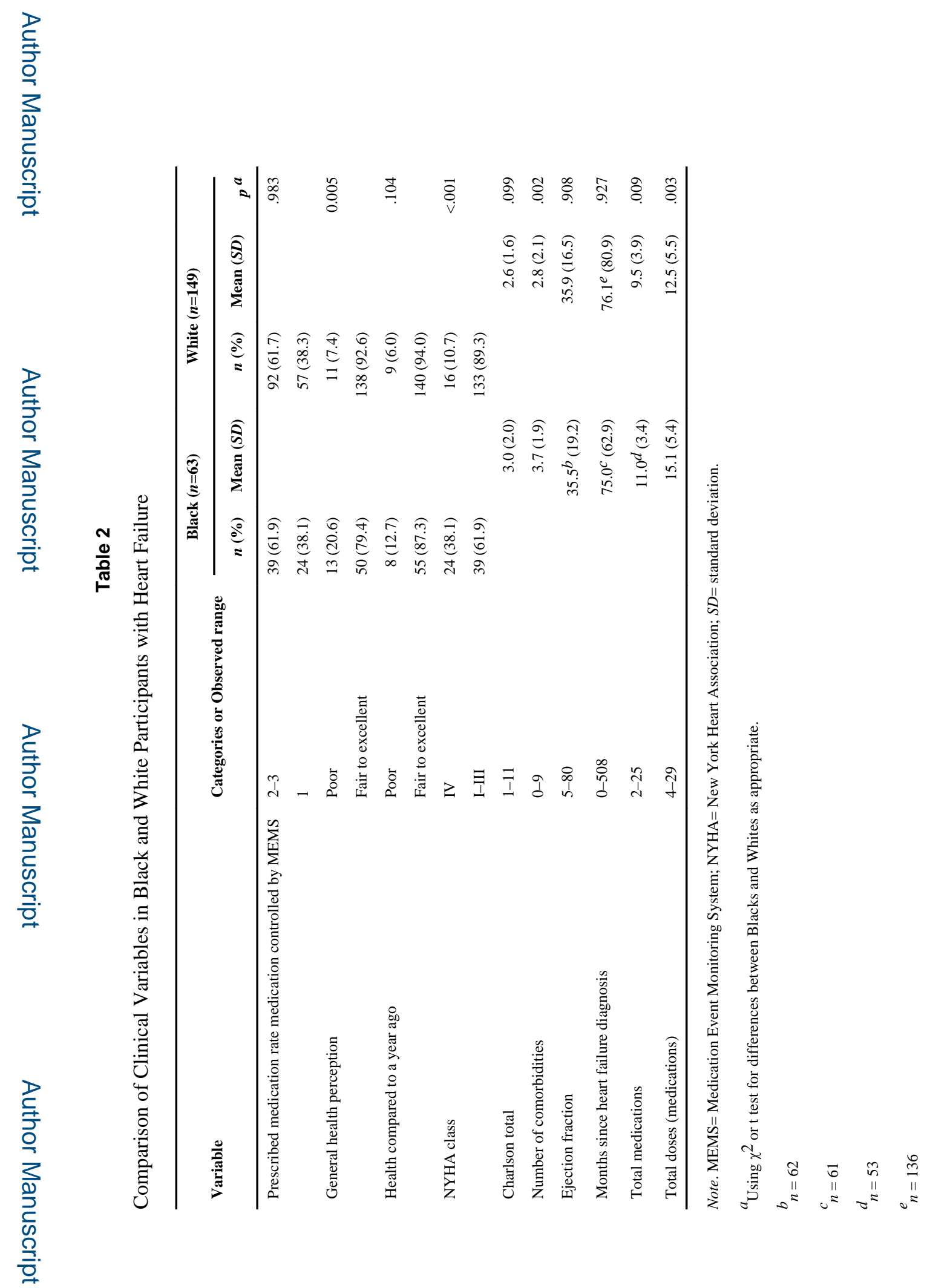

Res Nurs Health. Author manuscript; available in PMC 2016 August 01. 


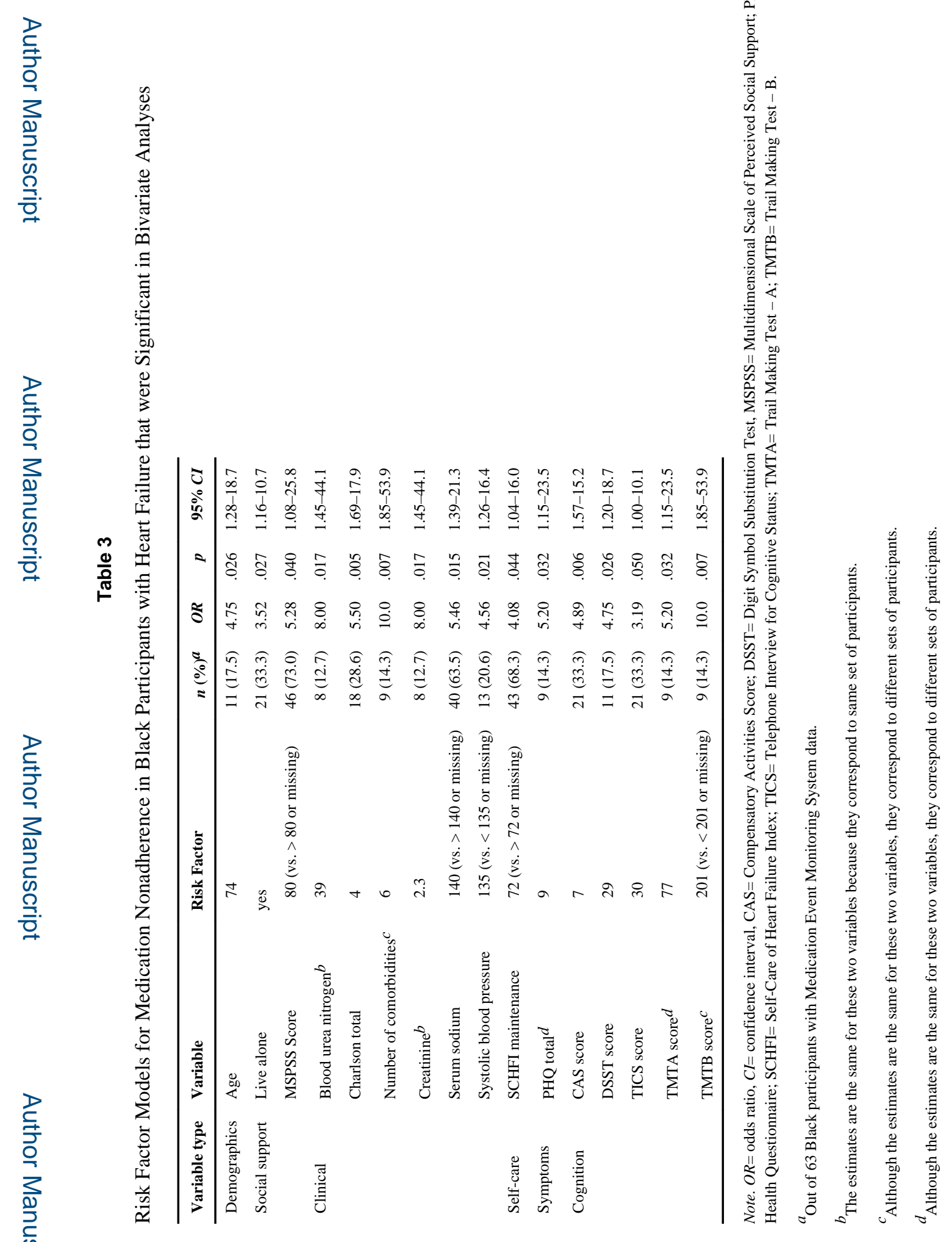




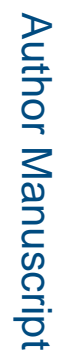

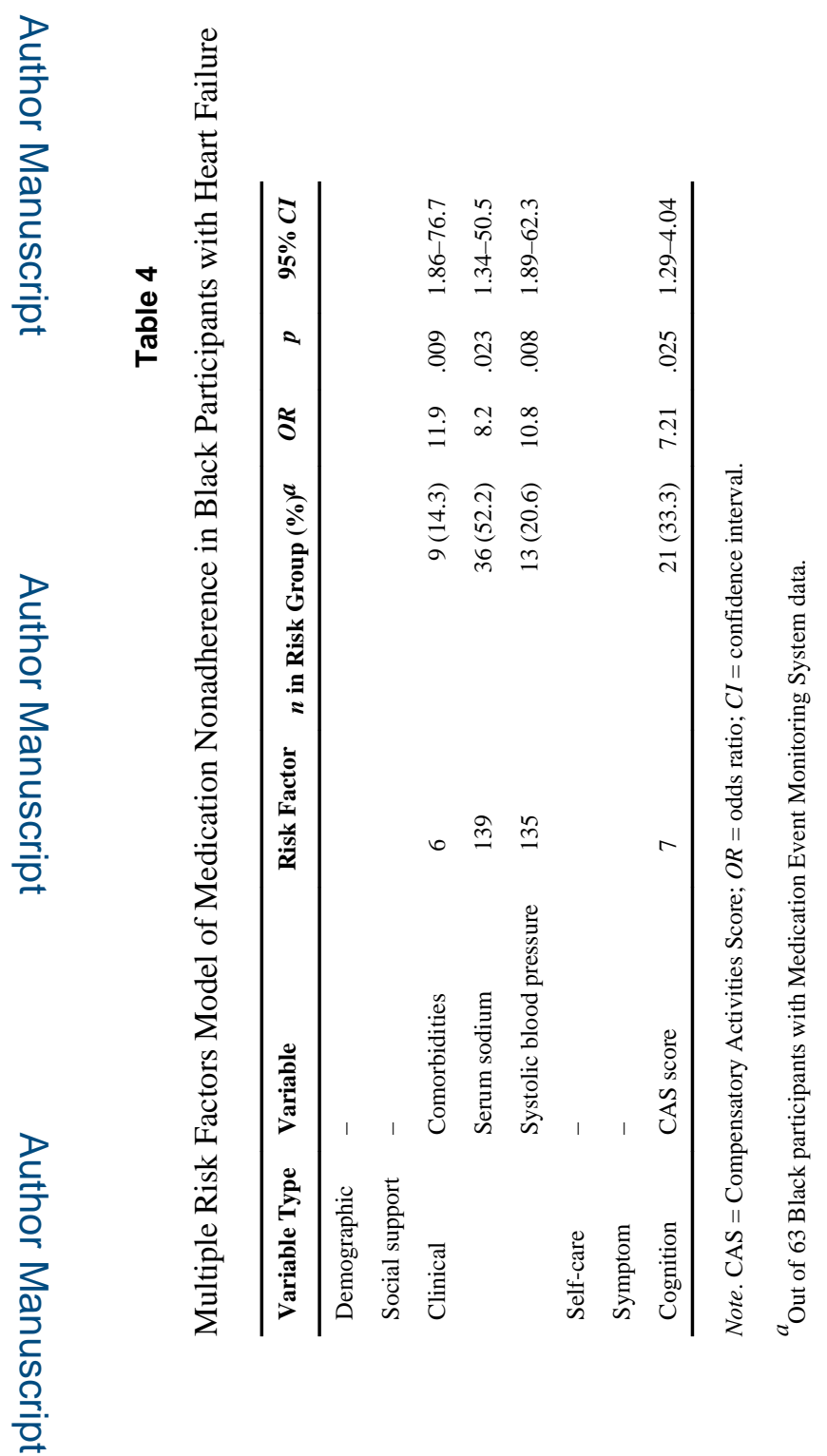

Res Nurs Health. Author manuscript; available in PMC 2016 August 01. 


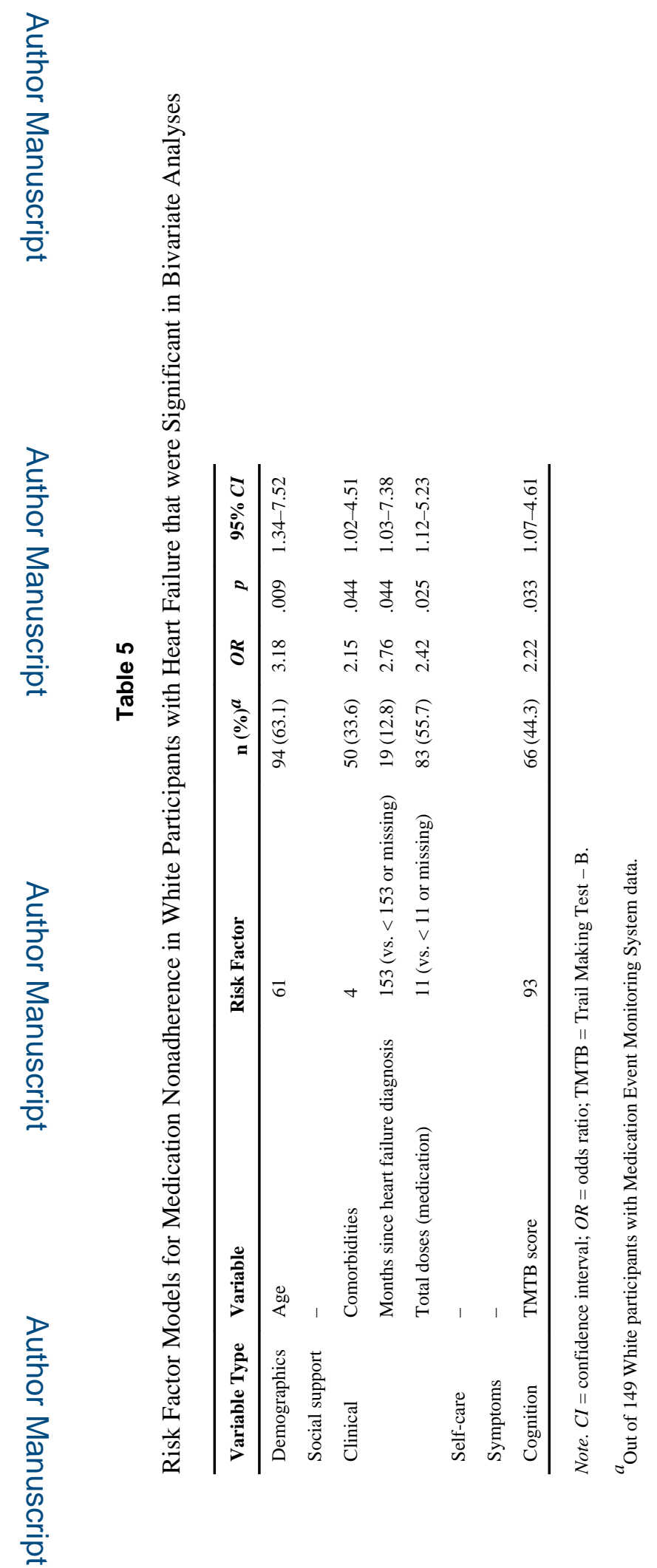

Res Nurs Health. Author manuscript; available in PMC 2016 August 01. 